\title{
Benford's law first significant digit and distribution distances for testing the reliability of financial reports in developing countries
}

\author{
Jing Shi* and Marcel Ausloos ${ }^{\dagger}$ \\ School of Business, Ken Edwards Building, \\ University of Leicester, Leicester, LE1 7RH, United Kingdom
}

Tingting $\mathrm{Zhu}^{\ddagger}$

Faculty of Business and Law, Hugh Aston Building, De Montfort University, Leicester, LE1 9BH, United Kingdom

December 4, 2017

\begin{abstract}
We discuss a common suspicion about reported financial data, in 10 industrial sectors of the 6 so called "main developing countries" over the time interval [2000-2014]. These data are examined through Benford's law first significant digit and through distribution distances tests.

It is shown that several visually anomalous data have to be a priori removed. Thereafter, the distributions much better follow the first digit significant law, indicating the usefulness of a Benford's law test from the research starting line. The same holds true for distance tests. A few outliers are pointed out.
\end{abstract}

${ }^{*}$ e-mail address: 248299825@qq.com

${ }^{\dagger}$ e-mail address: ma683@le.ac.uk; also at GRAPES, 483 rue de la belle jardinière, B-4031 Liège, Euroland; e-mail address: marcel.ausloos@ulg.ac.be;

${ }^{\ddagger} e$-mail address: tingting.zhu@dmu.ac.uk 
Keywords: Benford's law (BL); outliers; distributions distance to BL

\section{Introduction}

The Benford's law is a phenomenological law about the probability distribution of the first significant digits (henceforth FSD) in a data set [1]. Newcomb (in 1881) and later Benford (in 1938) [2, 3] observed that the occurrence of the first significant digit in many data sets is not uniform, but tends to follow a logarithmic distribution such that the smaller digits appear as a first significant digit more frequently than the larger ones, according to

$$
N_{d}=N \log _{10}\left(1+\frac{1}{d}\right), \quad d \equiv 1,2,3, \ldots, 9
$$

where $N$ is the total number of considered 1-st digits, in short, the number of data points, and $N_{d}$ is the number of the so observed integer $d(=1,2,3, \ldots, 9)$ being the starting one (1-st) in the data set list [4].

The law, Eq. (1), nowadays called Benford's law (BL), is widely applied in the investigation of data manipulation by researchers in finance and economy [5, 6, 7]. The BL can be used not only to identify falsely created data, e.g. in corporations financial statements as shown by Nigrini [8], but also to verify the (non)reliability of macroeconomic data [9]. Furthermore, because of its special features, BL has been employed as a quality test criterion for various employed data. An extensive bibliography, from 1881 up to 2006, on Benford's law papers including theories, applications, generalizations and warnings can be found in a Hürlimann unpublished work but available on internet [10]; see also books by Kossovsky for more recent reviews [11, 12].

In particular, the Benford's law test (henceforth BL test) has been applied to many financial numerical series. Concentrating on stock market indices, let us mention (in a chronological order) Ley (in 1996) [13] with a Bayesian approach observed the distribution of U.S. stock indexes' digits: the series of one-day returns, for the Dow Jones Industrial Average (DJIA) and the Standard and Poor's Index (S\&P), were found to follow Benford's Law. Ley interestingly concluded that distributions that follow Benford's Law are distributions where small changes are more likely to occur than large changes. The FTSE 100 was examined by De Ceuster et al. (in 1998) [14].

\footnotetext{
${ }^{1}$ Financial Times Security Exchange
} 
After about a 10 years lapse with no investigation of the sort, Krakar and Žgela (in 2009) studied the Zagreb Stock Exchange and found out that the closing prices did not follow BL [15].

Corazza, et al. (in 2010) checked the S\&P 500 [16], while Zhao and Wu (also in 2010) wondered whether the Shanghai Stock Exchange Composite index and the Shenzhen Stock Exchange Component index agree with Benford's Law, finding that Benford's law reasonably holds for these two (main) Chinese stock indices [17.

Žgela (in 2011) analysis of DAX ${ }^{2}$ percentage changes over 10 years [20012011] led to the conclusion that DAX values were not in accordance with the FSD Law [18]. More recently, the Istanbul Stock Exchange (BIST) attracted some attention: Karavardar (in 2014) [19] found no disagreement with BL for the monthly returns (over 26 years), while Cinko (2014) found no disagreement for the daily returns (over 23 years) [20].

Under the efficient market hypothesis, the stock price index should be completely controlled by the market, thus would not be manipulated by human (or government) intervention. Under this situation, we could use the BL test to detect if faults exist in the price indexes

For completeness, let us mention opinions wondering whether BL has to be ever applied, e.g. as discussed by Mebane [5] or Durtschi et al. [21]. An argument on the non-reliability of detecting fraud by BL is based on the knowledge of BL by potential swindlers, aware of the necessity of conforming to the BL. In fact, such a possibility might not be surprising, or is even sustained, in view of findings on income taxes regularity in some Italian provinces by Mir et al. [7, 22]. Such an argument suggests to consider alternative methods complementing the BL classical analysis [23, 24, 25].

We propose here a data aggregation method (see also Mir et al. [7]) which could prove to be useful, especially in view of the fact that it would be particularly difficult to fabricate data conforming to BL by various unconnected agents along this scheme.

We have conducted the BL test in order to evaluate whether the collected Financial Times Security Exchange (henceforth FTSE) global price index of some financial data possibly contains error values. We have examined the case of monthly log-returns for six (top, - from a Gross Domestic Product (GDP) point of view) emerging countries, over 10 different industrial sectors, - over 15 recent years, as reported by FTSE. There is no study to

\footnotetext{
${ }^{2}$ Deutscher Aktien IndeX
} 
our knowledge, about data from emerging (or developing) countries, on the scale endeavored here below, but we mention that the GDP growth rate was studied for Germany by Gunnel and Tödter, in 2009, [26].

Therefore, the present paper goes as follows: after quickly outlining the BL test, in Sect. 2.1, we complement the methodology introducing a "complementary distance study" 2.2. In Sect. 3, after explaining the countries selection (Sect. 3.1), the raw data acquisition is recalled, i.e. monthly logreturns, next aggregated over 6 relevant emerging countries for a 15 year time interval (Sect. 3.2). The data of interest is displayed through histograms in Sect. 3.3 .

We have performed the $\chi^{2}$ tests in order to compare the BL to the observed distributions. The results appear unsatisfactory. However, a visual review indicates anomalous values in the raw data. We have removed them and redone $\chi^{2}$ tests with respect to BL test values; a comparison of results before and after removal of anomalous data is presented in Sect. 3.4.

A discussion, practically oriented on BL and on the distribution distance to BL is found in Sect. 4.1. In addition, since we also investigate a complementary quantity, the distance between the 10 industry price indexes FSD distributions and BL is discussed in Sect. 4.2. Some synthesis allows for a discussion in Sect. 5. Sect. 6 serves for a conclusion.

\section{Methodology}

\subsection{Benford's law}

Benford's law, Eq.(1), is known as the first digit law or the law of the leading digits. According to Eq.(1), in a given data set the probability of occurrence of a certain digit as the first (1-st) significant digit decreases logarithmically as the value of the digit increases from 1 to 9 . Thus, digit 1 should appear as the first significant digit about $30.10 \%$ times, and similarly 9 should appear about $4.58 \%$ times.

Benford's law, Eq. (1), holds for data sets in which the occurrence of

numbers is free from any restriction; significant deviations from the Benford distribution may indicate fraudulent or corrupted data [27].

Deviation from the expected Benford's law distribution is calculated from 
the $\chi^{2}$ statistic according to

$$
\chi_{1 B L}^{2}=\sum_{i=1}^{9} \frac{\left(e_{i 1}-b_{i 1 B}\right)^{2}}{b_{i 1 B}},
$$

where $e_{i 1}$ is the observed frequency of each FSD in the price index data; $b_{i 1 B}$ is the frequency expected from Benford's law. The $10 \%, 5 \%$, and $1 \%$ critical values for $\chi^{2}$, with 8 degrees of freedom, are 13.36, 15.51, and 20.09.

In practice, applications of Benford's law for fraud detection routinely may use more than the first digit [28]. Indeed, Eq. (1) can be generalized to forecast how many times any digit, or any combination of digits, should be found at some rank in the number [29]. Moreover, undetectable correlations by means of the classical covariance-based measure can be identified in the statistics of the corresponding first digits, as recently shown by Gramm et al. [30. We will not go beyond the FSD in this report, - going beyond seems indeed that we would mean to pursue a deleterious way on the data; this is outside our aim.

\subsection{Distribution distance}

The question on reliability of data along BL can be debated on theoretical grounds as mentioned here above [5, 6, 21]. In order to complement such a study we propose to examine another measure, - the distance between distributions as discussed by Cho and Gaines or by Miskiewicz [31, 32]. A distance measure can be constructed as the Euclidean distance between two distributions: this distance measure $d^{*}$ is defined as

$$
d^{*}=\frac{1}{M} \sqrt{\sum_{i=1}^{9}\left(e_{i 1}-b_{i 1 B}\right)^{2}},
$$

where $M$ is the maximum possible distance

$$
M=\operatorname{Max}_{i=1, \ldots, 9}\left[\left|e_{i 1}-b_{i 1 B}\right|\right] .
$$

We also introduce a distance measure $a^{*}$, as the absolute value of the difference between the mean of the investigated FSD distribution and the mean of BL FSD distribution divided by the maximum possible difference. 
It is worth mentioning that high values of $\chi^{2}, M, d^{*}$, and $a^{*}$ indicate weak similarities between the tested distribution and the theoretical BL distribution, while a large correlation coefficient reflects that the data FSD distribution is rather similar to the Benford's Law distribution.

\section{Data set}

\subsection{Country selection}

To perform an interesting selection, several emerging countries nominal GDP for 2014 were first looked at. The GDP data for the countries which we examined can be taken from the International Monetary Fund's World Economic Outlook Database 2015 [33. It is found that the nominal GDP of China, Brazil, India, Russia, Mexico, Indonesia, Turkey and Saudi Arabia are all larger than six hundred millions USD, i.e. those countries nominal GDPs rank higher than all other developing countries. Therefore, each of these eight countries economic power is comparatively stronger than that of any other developing countries. Thus, those countries financial data appear to provide a valuable representativeness for the whole set of developing country economic sectors. However, we could not take Russia and Saudi Arabia into account due to some data unavailability; we could not find suitable proxies to reflect industries returns.

\subsection{Financial data}

Recall that we implement a test on the reliability of (log-return) values obtained through aggregated data from emerging countries across various industry sectors.

The benchmark, which might be the most acceptable industry classification approach, divides all traded equities into ten different industry categories. We follow the FTSE industry classification: basic materials (MATS), consumer goods (GDS), consumer services (SVS), financials (FIN), health care (HEA), industrials (INDU), oil and gas (OIL), technology (TECH), telecommunications (TELE), and utilities (UTIL). All 10 industries sections are investigated in this research. In order to measure each industry stock return, we employ the FTSE Global monthly price index (http : //www.ftse.com/products/indices/geis - series). We transfer them into 
log returns. All the return rates are reported as percentages.

It is also worth pointing that there are some (known or not) limitations in the FTSE index. First, it only employs some specific portfolios to represent the whole market. Second, the FSTE price index is not available for several countries' specific industry sections. For example, there is no FSTE price index for Mexico's technology industry. Because of those reasons, we limited the selection to China, Brazil, India, Mexico, Indonesia and Turkey. The number of data points $N_{s}$ depends on the sector $s$.

We also chose monthly data as our relevant set for the following reasons: on one hand, monthly data is most frequently used in (related) previous studies; on the other hand, monthly data is more available than daily or quarterly data because numerous statistics departments employ the month as the time interval in their comparative measures, are easier to manipulate in view of identifying changes in trends, and better for strategic long term forecasting.

All data used in this research was downloaded from Datastream. For example, we collected data of FTSE China basic material (MATS) price index, and computed the monthly log return to represent the return of China's basic material industry. We did so for the 6 countries and the various industry sectors for the January 2010 - December 2014 time span. For uniformity, the currency unit of the collected price indexes was turned into U.S. dollar. In so doing we have a coherently aggregated data set on which BL tests can be performed.

\subsection{Histograms of raw data}

After collecting the data for the six countries monthly log returns in the 10 sectors for the time span[Jan. 2000 - Dec. 2014], one can draw the histograms of the dataset. Figure 1 displays the histograms of the 10 industries monthly log return aggregated over countries and the considered time interval. The descriptive statistics of data is found in Table 1 .

Note that the raw data extends over several orders of magnitudes. For example, the spreads [Max - min] extend between $\sim 55$ (for UTIL) to $\sim 300$ (for SVS). Industries, such as consumer services (SVS) and oil and gas (OIL), have maximum returns larger than 100 percent. Moreover, the minimum return of consumer services (SVS) is -105.8 percent, - which is smaller than -100 percent. The value range of industry returns standard deviations is wide: $\sim 6$, and even $\sim 9.5$ for SVS. Notice that it practically means that the 
return of this industry is less stable than that of others within the research period. Those phenomena are uncommon for stock returns. Since there are several abnormal statistical features the histograms of those industry returns have relatively high peaks and thin tails. The coefficient of variation (StdDev/mean) extends between 11 for GDS up to about $\sim 300$ for TECH.

\subsection{Histograms of adapted data}

As anticipated, abnormal repetitious values exist in the raw data. An example, - extracted from the whole data set, is provided in Table 2, covering relevant information on China 10 sectors between June 2000 and May 2001. This example visually demonstrates abnormal repetitions in the data set. They are emphasized with bold font. The table blanks also show some unavailable data. No certified interpretation of such repetitions could be found. We suspect that those repetitious numbers are error values due to technical problems of Datastream. Consequently, we checked the daily data of the same price indexes in the same period to justify our speculation. We observed that repetitions still exist in the same time periods. Hence, those values which would generate a biased result of the empirical test have been deleted. The descriptive statistics of such "adapted data" is found in Table

3. Figure 2 displays the corresponding histograms of the adapted monthly log return for the 10 industries, aggregated over the 6 countries, and for the considered 15 years time interval.

After such a data manipulation, the distribution characteristics are unchanged for MATS and quasi unchanged for TECH. However, the spreads [Max - min] extend now between $\sim 36$ for HEA to $\sim 82$ for SVS. Industries, the maximum returns are not larger than 45 percent (for TECH). Moreover, the minimum return of consumer services (SVS) is $-62 \%$ now. The value range of industry returns standard deviations is narrowed: as "low" as $\sim 4.3$ for HEA, and as "high" as $\sim 6$ for TECH and MATS. The coefficient of variation extends between $\sim-230$ (for SVS, for which the mean takes a negative value), to about $\sim 245$ (for $\mathrm{TECH})$.

In order to emphasize our data manipulation rationality, we have conducted the BL and distance tests on the adjusted data, but also on the raw FTSE aggregated price indexes. 


\section{Benford law test and distance test}

Thereafter, the data can be analyzed along BL tests through a $\chi^{2}$ as follows.

Figure 3 illustrates the 10 industry price indexes FSD frequencies and the expected frequency according to BL. We drew those bar charts with the frequencies reported in Table 4. The somber (red) bars represent the observed distributions, the dark (blue) bars represent the Benford's law theoretical distribution. The bar charts show that all industries FSD distribution are different from the expected BL FSD distribution.

Beside that, Figure 4 illustrates the same type of data, but after the deletion of the abnormal repetitious numbers; the frequencies are reported in Table 5. The somber (red) bars again represent the observed distributions, the dark (blue) bars represent the BL expectation. The bar charts shows that all industries FSD distribution are still different from BLFSD distribution. However, comparing to the distributions before adjustment, those distributions are more similar to the expected BL distribution.

\subsection{Goodness of fit test analysis}

How much the distributions of the first digits match the distribution specified by BL, Eq.(2), can be now tested through a $\chi^{2}$ test. The results for the raw data and for the adapted data are found in Table 6 and Table 7 , respectively. The $^{* *}$ indicates a $99 \%$ significant departure from BL.

Comparing Table 6 and Table 7 , it is found that all $\chi^{2}$ coefficients are large. However, almost all investigated FSD distributions become similar to the BL distribution shown in Table 7. Thus, the data set statistical quality becomes better after the deletion of abnormal value repetitions.

Nevertheless, exceptionally, the BL $\chi^{2}$ test shows that the FSD distribution of financial (FIN) price index becomes less similar to the BL distribution after adjustment. However, since the differences are very small, and deliver the lowest $\chi^{2}(\sim 25)$, the deletion of the abnormal values of the financial price index nevertheless leads to a more appealing (or reliable) financial data set rationality.

\subsection{Distance analysis}

Distance measures are found to be smaller when anomalous repetitions are

removed. In addition, the high values of $d^{*}$ and $a^{*}$ show that the adapted 
distributions are consistently more similar to the BL distribution.

Notice that the $a^{*}$ of HEA seems to be an outlier, both before $(\sim 0.28)$ and after $(\sim 0.22)$ removing anomalous values. This is in contrast to the $a^{*}$ TECH value, which is an outlier in the raw data analysis, but turns out to be "very reasonable", after removing anomalous values.

\section{Discussion}

The present work has two aims. One is a test of Benford's law on specific data, which addresses the question whether the aggregated data method is reliable; the second aim is to touch upon the question whether Benford's law applies to aggregated data, in particular from emerging countries for various industry sectors. An answer to the latter question is relevant before producing any research activity on comparing, for example, investments, growth, and financial indices of various sectors before economic policy or portfolio strategies.

It has been argued that it is of interest to analyze such financial data along BL lines. It is much agreed with e.g. Judge and Schechter [6] that BL is definitively not an indubitable test of data exactness. Thus, some deviation from the Benford distribution would not provide a conclusive proof of " data manipulation", just as conformity does not prove cleanliness of the data. Nevertheless, BL may be considered useful as an aid in analytical procedures of testing the exactness of financial reports [27].

Thus, if the test is conclusive, analyzers would be happy, but if not, this induces more questions and reflexions. The apparent lack of agreement with BL for the 1-st digit of the raw data only, as found in the previous sections is somewhat frightening. Fortunately, observing the existence of anomalous values leads to a more agreeable aspect of the data. Therefore one can conclude that the BL test in Sect; 4 was useful on the original data.

This leads to basically two sets of questions, economic and financial ones, about the specificity of the aim, resulting from an accumulation of items:

- (i) a first criticism should be on the aggregation method; one could demand more information on the items leading to the final sum of values. One can indeed wonder about what was really accounted for? Although the reported values in each country pertained to a concluded year, and might concern different items, it might occur that some rounding factor accumulated so much in a few cases as modifying the first digits 
of items and finally the global report. Our argument is that we have examined such a possibility and took care as much as possible of a coherent scheme.

- (ii) The anomalies might be only the result of sloppy, deliberate manipulation or unintentional but lazy accounting, quite in contrast from data manipulation by Governments [9]. We have indeed considered such a possibility, and removed suspicious data.

- (iii) A third hypothesis might have a more fundamental aspect: indeed, non-conformity with Benford's law should not be qualified as a reliable sign of poor quality of macroeconomic data, but could rather be based on marked structural shifts in the data set [34. Gonzales-Garcia and Pastor point out that (we quote) "rejection of Benford's law may be unrelated to the quality of statistics, .... . Hence, nonconformity with Benford's law should not be interpreted as a reliable indication of poor quality in macroeconomic data". In some sense, this is a safe side confirming the need of a BL test before further research, and proposing a reflection on the data at the first analytical stage.

\section{Conclusions}

We have looked at 10 industry sectors, in 6 top emerging countries, over a 15 year time span. The econophysics analysis pertains to an accounting procedure along Benford's law first significant digit and to some statistical analysis of the distance between distributions. It is shown that several visually anomalous data have to be a priori removed.

One conclusion is that complementary accounting techniques tests should be considered before deciding whether some data is faked or erroneous. Moreover, the results indicate that data reliability is a mandatory aspect to be observed before proceeding with further analysis and modeling. Some practical information has to be necessarily outlined before and after some scientific analysis. 


\section{References}

[1] M. J. Nigrini, Benford's Law: Applications for forensic accounting, auditing, and fraud detections (John Wiley \& Sons, 2012).

[2] S. Newcomb, Note on the frequency of use of the different digits in natural numbers, Amer. J. Math. 4 (1881) 39-40.

[3] F. Benford, The law of anomalous numbers, Proc. Amer. Philos. Soc. 78 (1938) 551-572.

[4] A. Berger and T. P. Hill, A basic theory of Benford's Law, Probability Surveys 8 (2011) 1-126.

[5] W. R. Mebane Jr, 'Election forensics: the second-digit Benford's law test and recent American presidential elections.' in Election fraud: detecting and deterring electoral manipulation, (Brookings Institution Press Washington, DC, 2008), pp. 162-81

[6] G. Judge and L. Schechter, Detecting Problems in Survey Data Using Benford's Law, J. Hum. Resour. 44 (2009) 1-24.

[7] T. A. Mir, M. Ausloos, and R. Cerqueti, Benford's law predicted digit distribution of aggregated income taxes: the surprising conformity of Italian cities and regions. Eur. Phys. J. B 87 (2014) 261.

[8] M. J. Nigrini, Digital analysis using Benford's law: tests statistics for auditors, (Global Audit Publications, Vancouver, 2000)

[9] B. Rauch, M. Göttsche, G. Brähler, S. Engel, Fact and Fiction in EUGovernmental Economic Data, Germ. Econ. Rev. 12 (2011) 243-255.

[10] W. Hürlimann, Benford's law from 1881 to 2006, http : //arxiv.org/abs/math.ST/0607168 (2006)

[11] A.E. Kossovsky, Benford's Law: Theory, the General Law of Relative Quantities, and Forensic Fraud Detection Applications. (World Scientific Publishing Co, 2014.)

[12] A.E. Kossovsky, Small is Beautiful,

https : //www.amazon.com/Small - Beautiful - Numerous - Rare World/dp/069291241X (2017). 
[13] E. Ley, On the peculiar distribution of the U.S. stock indexes' digits. Amer. Statist. 50 (1996) 311-313.

[14] M. J. K. De Ceuster, G. Dhaene, and T. Schatteman, On the hypothesis of psychological barriers in stock markets and Benford's Law, Journal of Empirical Finance 5 (1998) 263-279 .

[15] Z. Krakar and M. Žgela, Evaluation of Benford's Law Application in Stock Prices and Stock Turnover, Informatologija 42 (2009) 158-165.

[16] M. Corazza, A. Ellero, and A. Zorzi, Checking financial markets via Benford's law: the S\&P 500 case, in Mathematical and statistical methods for actuarial sciences and financ, M. Corazza and C. Pizzi, Eds. (SpringerVerlag Italia, 2010) pp. 93-102.

[17] S. Zhao and W. Wu, Does Chinese Stock Indices Agree with Benford's Law? in Management and Service Science (MASS), 2010 International Conference on, pp. 1-3.

[18] M. Žgela, Application of Benford's law in analysis of DAX percentage changes, Cybernetics and Information Technologies 11 (2011) 53-70.

[19] A. Karavardar, Benford's Law and an Analysis in Istanbul Stock Exchange (BIST), International Journal of Business and Management 9 (2014) 160-172.

[20] M. Cinko, Testing distribution of BIST-100 returns by Benford Law, Journal of Economics Finance and Accounting 1 (2014) 184-191.

[21] C. Durtschi, W. Hillison, and C. Pacini, The effective use of Benford's law to assist in detecting fraud in accounting data, J. Forensic Acc. 5 (2004) $17-34$.

[22] M. Ausloos, R. Cerqueti, and T. A. Mir, Data science for assessing possible tax income manipulation: The case of Italy, Chaos, Solitons and Fractals 104 (2017) 238-256.

[23] M. J. Nigrini and S. J. Miller, Data diagnostics using second-order tests of Benford's law. Auditing: A Journal of Practice \& Theory 28 (2009) 305-324. 
[24] P. Clippe and M. Ausloos, Benford's law and Theil transform of financial data. Physica A: Statistical Mechanics and its Applications 39 (2012) 6556-6567.

[25] N. Gauvrit, J. Ch. Houillon, and J. P. Delahaye, Generalized Benford's Law as a Lie Detector. Advances in Cognitive Psychology 13 (2017) 121127.

[26] S. Gunnel and K. Tödter, Does Benford's Law Hold in Economic Research and Forecasting?, Empirica: Journal of Applied Economics and Economic Policy 36 (2009) 273-292.

[27] M.J. Nigrini and L.J. Mittermaier, The use of Benford's law as an aid in analytical procedures, Auditing-J. Pract. Th. 16 (1997) 52-67.

[28] M.J. Nigrini, A Taxpayer Compliance Application of Benford's Law, J. Amer. Tax Ass. 18 (1996) 72-91.

[29] T. A. Mir and M. Ausloos, Benford's law: a 'sleeping beauty' sleeping in the dirty pages of logarithmic tables, Journal of the Association for Information Science and Technology (2017). doi: 10.1002/asi.23845; http://arxiv.org/abs/1702.00554

[30] R. Gramm, J. Yost, Q. Su, and R. Grobe, 'Applications of the first digit law to measure correlations', Phys. Rev. E 95 (2017) 042136.

[31] W.K.T. Cho and B.J. Gaines, Breaking the (Benford) Law: Statistical Fraud Detection in Campaign Finance', The American Statistician 61 (2007) 218-223.

[32] J. Miśkiewicz, 'Entropy correlation distance method. The Euro introduction effect on the Consumer Price Index', Physica A: Statistical Mechanics and its Applications 389 (2010) 1677-1687.

[33] International Monetary Fund World Economic Outlook Database 2015 https : //www.imf.org/external/pubs/ft/weo/2015/01/weodata/index.aspx.

[34] J. Gonzales-Garcia and G. Pastor, Benford's Law and Macroeconomic Data Quality, International Monetary Fund, Working Paper, 2009 http://papers.ssrn.com/sol3/papers.cfm?abstract_id=135643 


\begin{tabular}{|c|c|c|c|c|c|}
\hline sector $s$ & $N_{s}$ & Mean & Std. Dev. & Min & Max \\
\hline MATS & 1080 & 0.288301 & 5.941751 & -35.97069 & 23.03240 \\
GDS & 1007 & 0.480227 & 5.265518 & -21.68574 & 80.21888 \\
SVS & 1080 & 0.242929 & 9.512246 & -105.81600 & 192.71100 \\
FIN & 1080 & 0.364663 & 5.282102 & -34.95486 & 22.45599 \\
HEA & 1060 & 0.270010 & 4.293489 & -17.68409 & 75.60660 \\
INDUS & 1080 & 0.289500 & 5.331192 & -34.29641 & 48.92794 \\
OIL & 963 & 0.189449 & 6.152058 & -28.31262 & 106.30770 \\
TECH & 563 & 0.018170 & 5.288614 & -25.36837 & 45.13081 \\
TELE & 1036 & 0.156854 & 5.138521 & -35.17884 & 62.35700 \\
UTIL & 914 & 0.278857 & 4.494412 & -28.60670 & 26.05142 \\
\hline
\end{tabular}

Table 1: Descriptive statistics of raw FTSE aggregated data distributions according to industrial and financial sectors

\begin{tabular}{|c|c|c|c|c|c|c|c|c|c|c|}
\hline Time & MATS & GDS & SVS & FIN & HEA & INDUS & OIL & TECH & TELE & UTI \\
\hline $19 / 06 / 00$ & 393.85 & 306.97 & 350.87 & 352.27 & 353.19 & 326.67 & & 324.88 & & 346.6 \\
$19 / 07 / 00$ & 419.54 & 318.72 & 375.72 & 390.77 & 392.12 & 332.86 & & 301.05 & & 372.6 \\
$19 / 08 / 00$ & 672.12 & 357.51 & 424.42 & 504.35 & 441.34 & 300.67 & & 342.92 & & 475.3 \\
$19 / 09 / 00$ & 551.57 & 323.42 & 316.22 & 359.97 & $\mathbf{4 4 4 . 1 9}$ & 232.00 & 345.30 & 315.77 & & 404.8 \\
$19 / 10 / 00$ & 436.36 & 263.26 & 291.00 & 333.02 & $\mathbf{4 4 4 . 1 9}$ & 217.52 & 316.80 & 236.61 & & 387.3 \\
$19 / 11 / 00$ & 514.74 & 318.45 & 349.38 & 440.82 & $\mathbf{4 4 4 . 1 9}$ & 254.57 & 374.48 & 323.73 & & 468.5 \\
$19 / 12 / 00$ & 483.85 & 301.04 & 341.78 & 469.70 & $\mathbf{4 4 4 . 1 9}$ & 253.05 & 330.55 & 309.58 & & 472.6 \\
$19 / 01 / 01$ & 494.17 & 276.96 & 365.06 & 494.72 & $\mathbf{4 4 4 . 1 9}$ & 253.83 & 351.20 & 307.70 & & 490.0 \\
$19 / 02 / 01$ & 571.19 & 293.50 & 392.49 & 476.44 & $\mathbf{4 4 4 . 1 9}$ & 256.90 & 423.46 & 277.65 & & 560.5 \\
$19 / 03 / 01$ & 641.28 & 496.10 & 410.26 & 661.23 & $\mathbf{4 4 4 . 1 9}$ & 395.62 & 436.04 & 337.65 & & 701.2 \\
$19 / 04 / 01$ & 796.57 & 638.30 & 464.53 & 934.58 & 665.35 & 508.53 & 465.78 & 425.80 & & 792.8 \\
$19 / 05 / 01$ & 837.03 & 686.18 & 512.03 & 989.45 & 643.00 & 561.10 & 543.03 & 425.03 & & 849.4 \\
\hline
\end{tabular}

Table 2: An extracted part of the whole data set. This table covers relevant information only on China between June 2000 and May 2001, from Datastream. We provide this table as an example to visually demonstrate abnormal repetitions in the data set. They are emphasized with bold font. The Table blank content also allows to point out that some data is sometimes unavailable. 


\begin{tabular}{|c|c|c|c|c|c|}
\hline sector $s$ & $N_{s}$ & Mean & Std. Dev. & Min & Max \\
\hline MATS & 1080 & 0.288301 & 5.941751 & -35.97069 & 23.03240 \\
GDS & 906 & 0.437259 & 4.870018 & -21.68574 & 24.15624 \\
SVS & 992 & -0.023972 & 5.527875 & -62.08636 & 19.45960 \\
FIN & 1075 & 0.398876 & 5.183369 & -26.34447 & 22.45599 \\
HEA & 724 & 0.266428 & 4.324815 & -17.68409 & 18.92318 \\
INDUS & 1007 & 0.261899 & 5.303363 & -34.29641 & 21.00709 \\
OIL & 816 & 0.051542 & 5.376211 & -28.31262 & 20.92057 \\
TECH & 449 & 0.024091 & 5.923321 & -25.36837 & 45.13081 \\
TELE & 1015 & 0.098663 & 4.809565 & -35.17884 & 22.98440 \\
UTIL & 745 & 0.375603 & 4.860502 & -19.12928 & 26.051420 \\
\hline
\end{tabular}

Table 3: Descriptive statistics of variables after the deletion of abnormal repetitious numbers. The variables are the 10 industries aggregated monthly stock return of the six countries mentioned in the text. As the table shows, the numbers of observations $N_{s}$ have been decreased, with respect to Table 1 data.

\begin{tabular}{|c|c|c|c|c|c|c|c|c|c|c|}
\hline sector $s$ & Obs & 1 & 2 & 3 & 4 & 5 & 6 & 7 & 8 & \\
\hline MATS & 1086 & 23.2044 & 18.3241 & 17.9558 & 13.2597 & 8.6556 & 5.5249 & 4.8803 & 4.4199 & 3 \\
GDS & 1013 & 35.5380 & 14.1165 & 7.5025 & 8.1935 & 12.4383 & 4.7384 & 5.4294 & 6.1204 & 5 \\
SVS & 1086 & 33.0571 & 14.5488 & 10.9576 & 7.7348 & 12.2468 & 5.5249 & 5.9853 & 6.9061 & 3 \\
FIN & 1086 & 36.2799 & 24.1252 & 9.0239 & 4.6961 & 3.9595 & 5.8932 & 6.2615 & 4.9724 & 4 \\
HEA & 1066 & 21.8574 & 4.2214 & 9.5685 & 9.0056 & 9.1932 & 7.4109 & 12.2889 & 10.9756 & 1 \\
INDUS & 1086 & 20.9024 & 17.6796 & 22.5599 & 4.7882 & 5.2486 & 9.1160 & 10.4052 & 5.3407 & 3 \\
OIL & 969 & 23.4262 & 26.4190 & 12.6935 & 8.5655 & 8.2559 & 4.8504 & 8.3591 & 3.7152 & 3 \\
TECH & 568 & 41.7254 & 16.0211 & 11.9718 & 11.6197 & 9.1549 & 4.5775 & 1.7606 & 1.4085 & 1 \\
TELE & 1042 & 39.4434 & 15.6430 & 4.7985 & 5.5662 & 6.1420 & 4.9904 & 7.2937 & 8.6372 & 7 \\
UTIL & 920 & 32.5000 & 9.8913 & 8.0435 & 19.0217 & 4.8913 & 9.0217 & 4.5652 & 6.4130 & 5 \\
\hline FSD BL & & 30.1030 & 17.6091 & 12.4939 & 9.6910 & 7.9181 & 6.6947 & 5.7992 & 5.1153 & 4 \\
\hline
\end{tabular}

Table 4: First significant digit (FSD) frequency of FTSE global price index for each industry and for each digit; the theoretically expected FSD value from $\mathrm{BL}$ is also given. 


\begin{tabular}{|c|c|c|c|c|c|c|c|c|c|c|}
\hline sector $s$ & Obs & 1 & 2 & 3 & 4 & 5 & 6 & 7 & 8 & \\
\hline MATS & 1080 & 23.2044 & 18.3241 & 17.9558 & 13.2597 & 8.6556 & 5.5249 & 4.8803 & 4.4199 & 3 \\
GDS & 906 & 35.5380 & 14.1165 & 7.5025 & 8.1935 & 12.4383 & 4.7384 & 5.4294 & 6.1204 & 5 \\
SVS & 992 & 33.0571 & 14.5488 & 10.9576 & 7.7348 & 12.2468 & 5.5249 & 5.9853 & 6.9061 & 3 \\
FIN & 1075 & 36.2799 & 24.1252 & 9.0239 & 4.6961 & 3.9595 & 5.8932 & 6.2615 & 4.9724 & 4 \\
HEA & 724 & 21.8574 & 4.2214 & 9.5685 & 9.0056 & 9.1932 & 7.4109 & 12.2889 & 10.9756 & 15 \\
INDUS & 1007 & 20.9024 & 17.6796 & 22.5599 & 4.7882 & 5.2486 & 9.1160 & 10.4052 & 5.3407 & 3 \\
OIL & 816 & 23.4262 & 26.4190 & 12.6935 & 8.5655 & 8.2559 & 4.8504 & 8.3591 & 3.7152 & 3 \\
TECH & 449 & 41.7254 & 16.0211 & 11.9718 & 11.6197 & 9.1549 & 4.5775 & 1.7606 & 1.4085 & 1 \\
TELE & 1015 & 39.4434 & 15.6430 & 4.7985 & 5.5662 & 6.1420 & 4.9904 & 7.2937 & 8.6372 & 7 \\
UTIL & 745 & 32.5000 & 9.8913 & 8.0435 & 19.0217 & 4.8913 & 9.0217 & 4.5652 & 6.4130 & 5 \\
\hline FSD BL & & 30.1030 & 17.6091 & 12.4939 & 9.6910 & 7.9181 & 6.6947 & 5.7992 & 5.1153 & 4 \\
\hline
\end{tabular}

Table 5: First significant digit (FSD) frequency of FTSE global price index for each industry after the deletion of abnormal repetitious numbers; the expected FSD value according to $\mathrm{BL}$ is also given.

\begin{tabular}{|c|c|c|c|c|c|}
\hline sector $s$ & Corr. coeff. & $\chi^{2}$ & $M$ & $d^{*}$ & $a^{*}$ \\
\hline MATS & 0.9092 & $40.5876^{* *}$ & 0.06898 & 0.09054 & 0.00479 \\
GDS & 0.9383 & $57.4226^{* *}$ & 0.05435 & 0.09129 & 0.01066 \\
SVS & 0.9616 & $54.1127^{* *}$ & 0.04328 & 0.06584 & 0.00147 \\
FIN & 0.9614 & $25.1805^{* *}$ & 0.06516 & 0.10787 & 0.05716 \\
HEA & 0.4426 & $90.1797^{* *}$ & 0.13387 & 0.19834 & 0.27801 \\
INDUS & 0.7543 & $39.9240^{* *}$ & 0.10065 & 0.14564 & 0.05613 \\
OIL & 0.8765 & $41.2437^{* *}$ & 0.08809 & 0.10870 & 0.00605 \\
TECH & 0.9814 & $49.7493^{* *}$ & 0.11622 & 0.12685 & 0.13523 \\
TELE & 0.9113 & $39.6741^{* *}$ & 0.09340 & 0.13053 & 0.02076 \\
UTIL & 0.8469 & $36.5188^{* *}$ & 0.09330 & 0.12873 & 0.03812 \\
\hline
\end{tabular}

Table 6: Pearson correlation coefficient, $\chi^{2}$ test value, the $M$ distance and other distance measures, as defined in the text, between BL and the investigated distributions; ** indicates a 99\% significant departure from BL. 


\begin{tabular}{|c|c|c|c|c|c|}
\hline sector $s$ & Corr. coeff. & $\chi^{2}$ & $M$ & $d^{*}$ & $a *$ \\
\hline MATS & 0.9092 & $40.5876^{* *}$ & 6.8986 & 0.0905 & 0.0048 \\
GDS & 0.9640 & $30.9417^{* *}$ & 5.6738 & 0.0748 & 0.0074 \\
SVS & 0.9723 & $38.0961^{* *}$ & 4.2938 & 0.0612 & 0.0035 \\
FIN & 0.9599 & $25.7035^{* *}$ & 6.6053 & 0.1103 & 0.0577 \\
HEA & 0.5483 & $78.9243^{* *}$ & 12.9368 & 0.1844 & 0.2168 \\
INDUS & 0.8550 & $35.4757^{* *}$ & 7.7164 & 0.1128 & 0.0657 \\
OIL & 0.9851 & $40.2773^{* *}$ & 2.5545 & 0.0395 & 0.0068 \\
TECH & 0.9304 & $56.5568^{* *}$ & 4.8145 & 0.0865 & 0.0598 \\
TELE & 0.9170 & $40.3456^{* *}$ & 10.1123 & 0.1341 & 0.0086 \\
UTIL & 0.9419 & $31.9800^{* *}$ & 9.6048 & 0.1125 & 0.0135 \\
\hline
\end{tabular}

Table 7: Pearson correlation coefficient, $\chi^{2}$ test value, the $M$ distance and other distance measures, as defined in the text, between BL and investigated distributions after data manipulation; ** indicates a $99 \%$ significant departure from BL. Comparing the distributions before and after adjustment through such coefficients shows that these distributions are more similar to the BL distribution, - except for the financial (FIN)'s FSD distribution. 

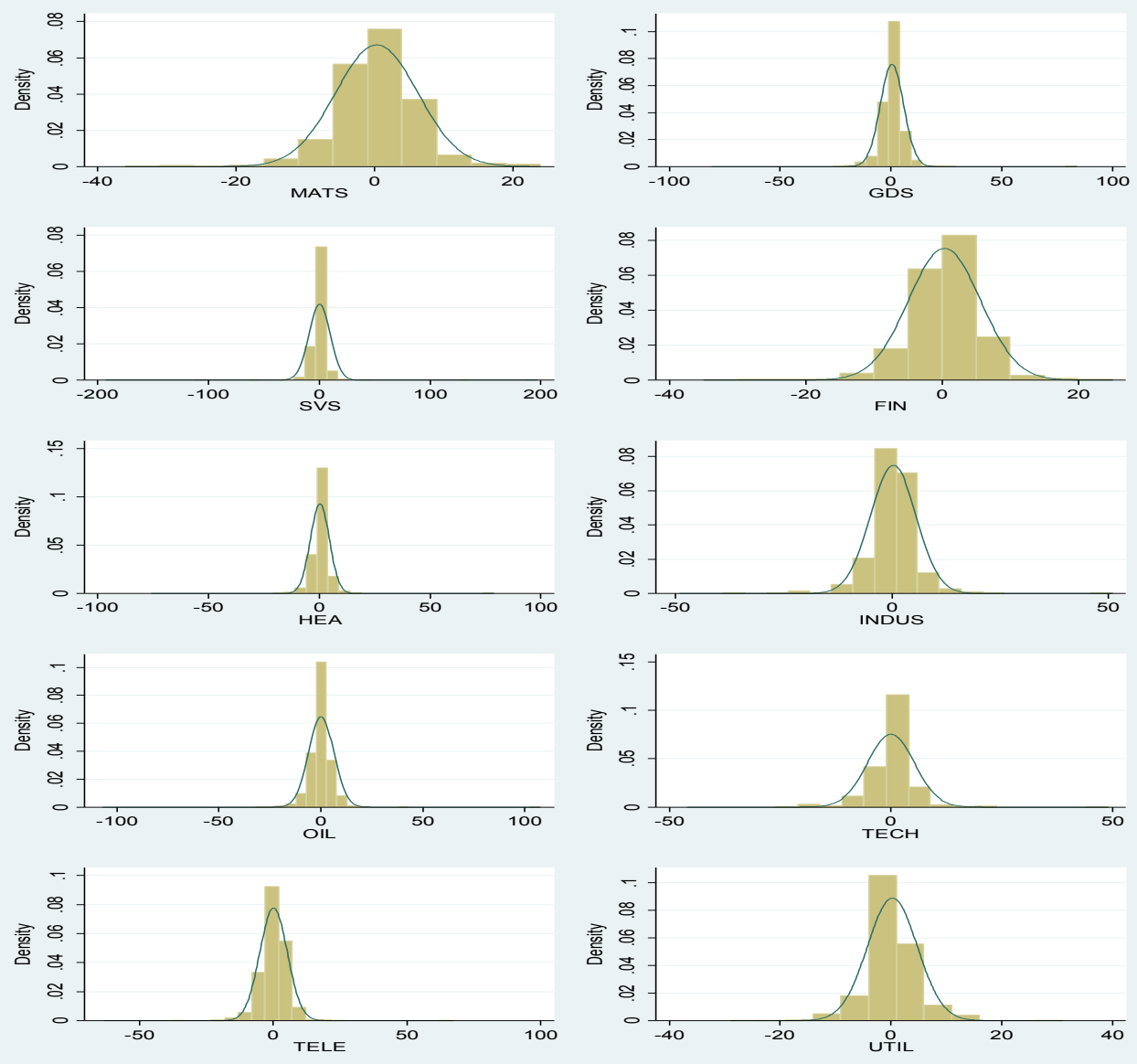

Figure 1: Distributions as histograms of the 10 industries monthly log return over 6 developing countries between January 2000 and December 2014. 

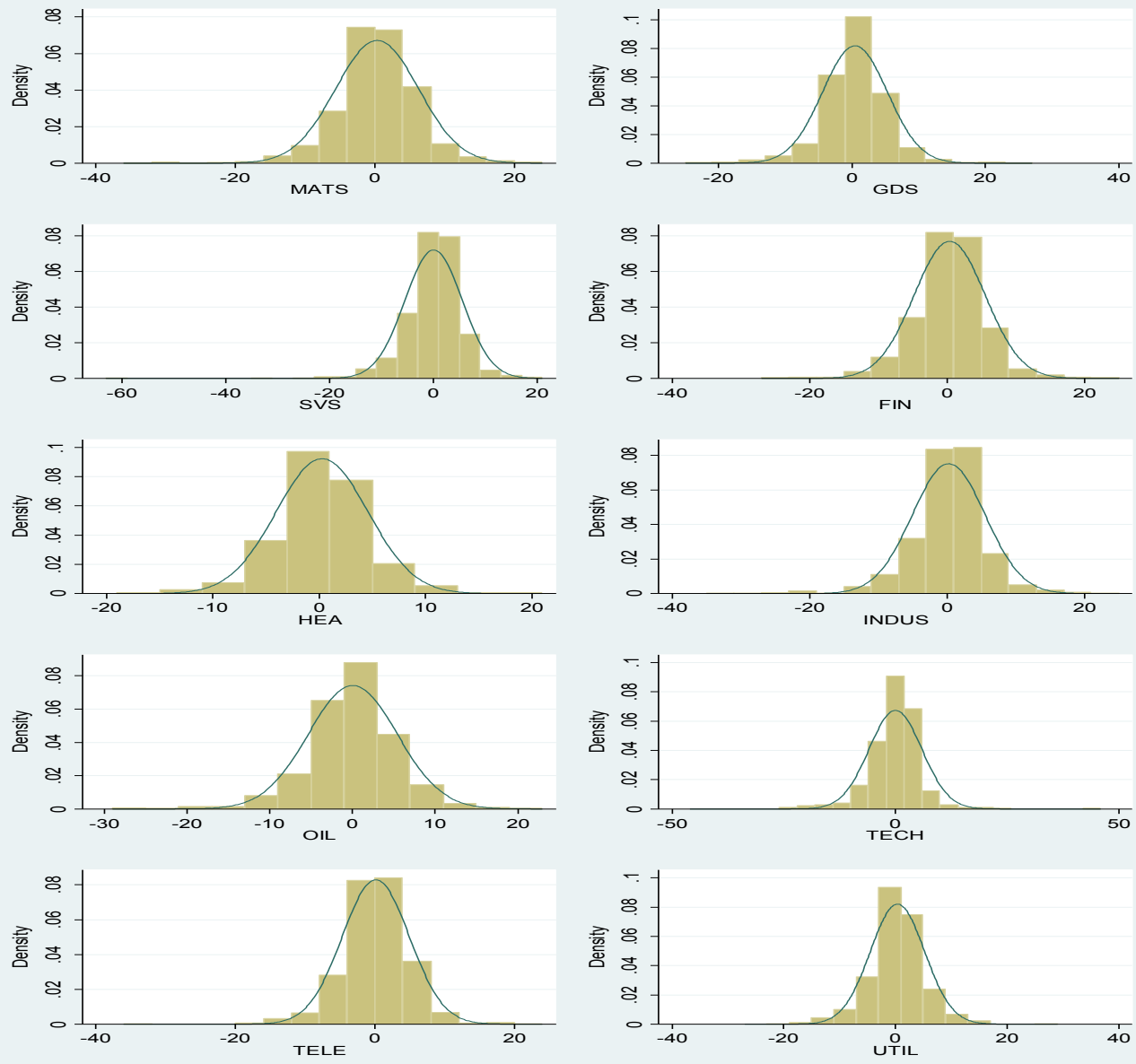

Figure 2: Histograms of the 10 industries' monthly log return distributions, after deletion of abnormal repetitious numbers, seen to be better fitted by a Gaussian 

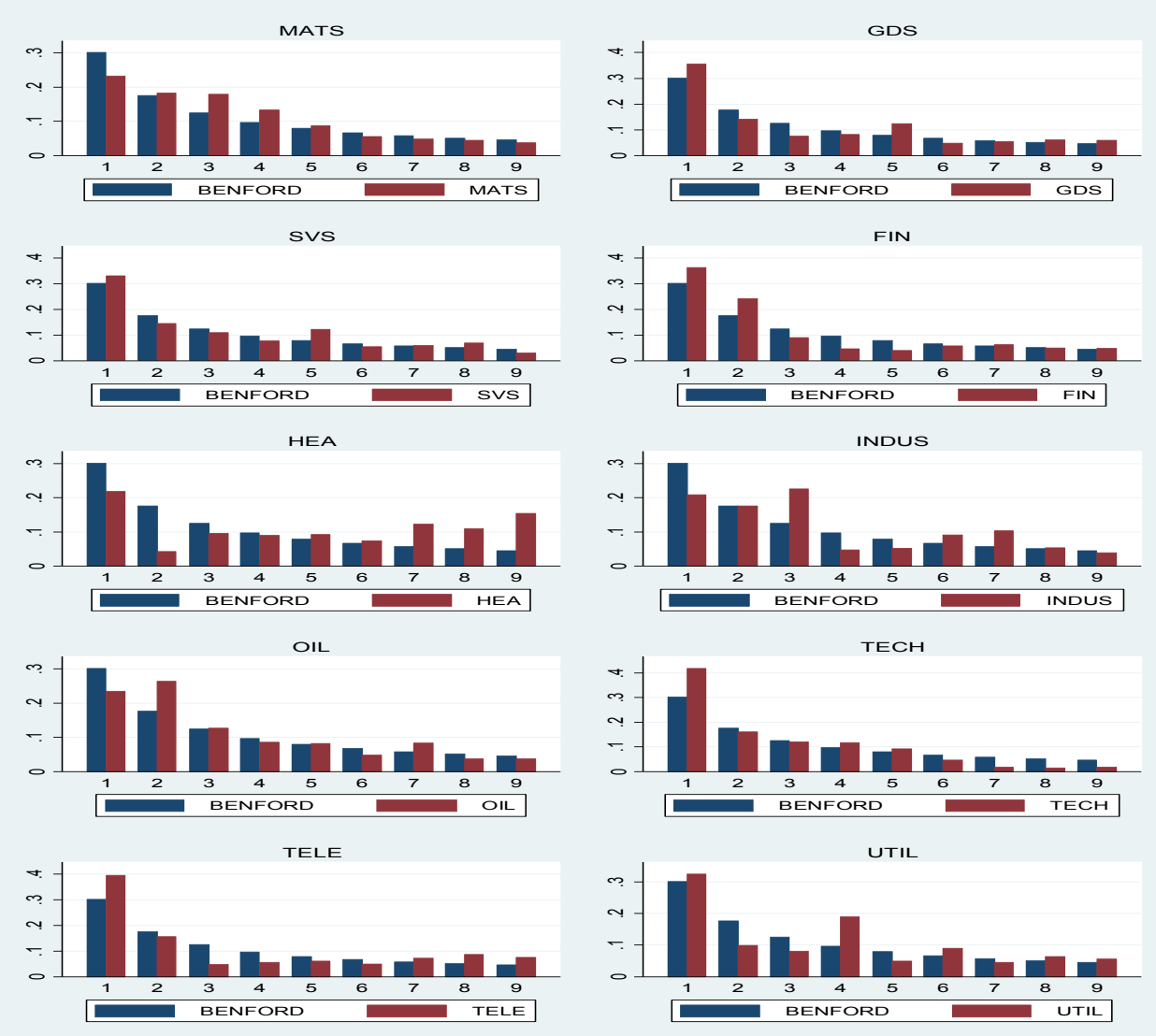

Figure 3: Comparison through bar charts of 10 industry price indexes FSD frequencies (red / light) and the expected frequency according to Benford's law (dark / blue) 

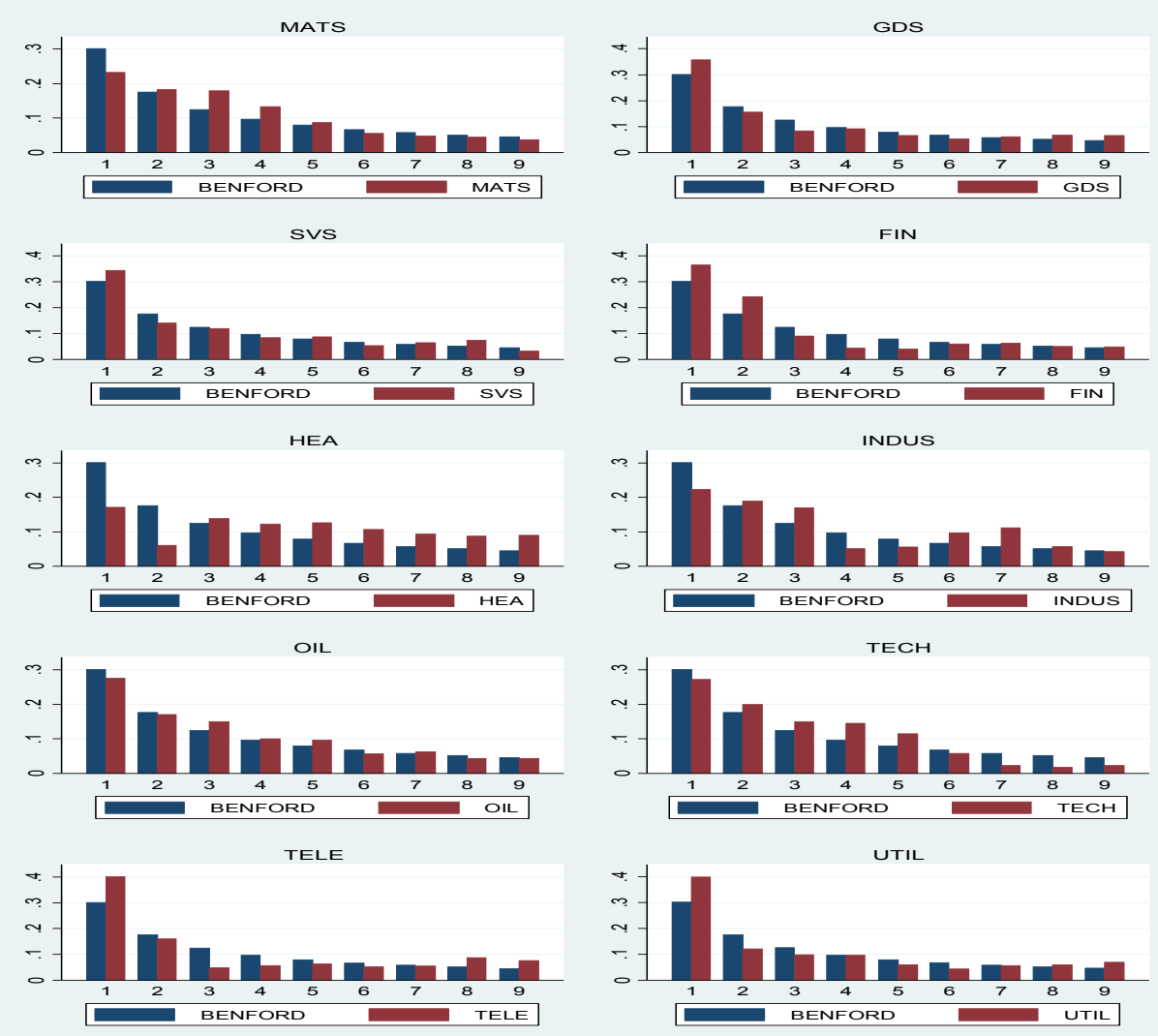

Figure 4: Comparison by bar charts of the 10 industry price indexes FSD frequencies (red / light) and the expected frequency according to Benford's law (dark / blue)after the deletion of abnormal repetitious numbers. 